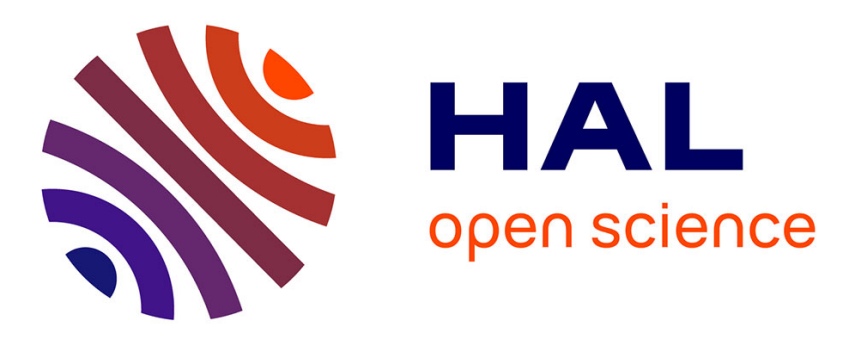

\title{
Epidemiology of borderline oxacillin-resistant Staphylococcus aureus in pediatric cystic fibrosis.
}

Timothy Ronan Leahy, Yvonne Yau, Eshetu Atenafu, Mary Corey, Felix Ratjen, Valerie Waters

\section{- To cite this version:}

Timothy Ronan Leahy, Yvonne Yau, Eshetu Atenafu, Mary Corey, Felix Ratjen, et al.. Epidemiology of borderline oxacillin-resistant Staphylococcus aureus in pediatric cystic fibrosis.. Pediatric Pulmonology, 2011, 10.1002/ppul.21383 . hal-00614855

\section{HAL Id: hal-00614855 https://hal.science/hal-00614855}

Submitted on 17 Aug 2011

HAL is a multi-disciplinary open access archive for the deposit and dissemination of scientific research documents, whether they are published or not. The documents may come from teaching and research institutions in France or abroad, or from public or private research centers.
L'archive ouverte pluridisciplinaire HAL, est destinée au dépôt et à la diffusion de documents scientifiques de niveau recherche, publiés ou non, émanant des établissements d'enseignement et de recherche français ou étrangers, des laboratoires publics ou privés. 


\section{Epidemiology of borderline oxacillin-resistant Staphylococcus aureus in pediatric cystic fibrosis.}

\begin{tabular}{|c|c|}
\hline Journal: & Pediatric Pulmonology \\
\hline Manuscript ID: & PPUL-10-0257.R1 \\
\hline Wiley - Manuscript type: & Original Article \\
\hline $\begin{array}{r}\text { Date Submitted by the } \\
\text { Author: }\end{array}$ & 19-Oct-2010 \\
\hline Complete List of Authors: & $\begin{array}{l}\text { leahy, timothy; Institute of Molecular Medicine, Genomic Research } \\
\text { Laboratory } \\
\text { Yau, Yvonne; The Hospital for Sick Children, Paediatric Laboratory } \\
\text { Medicine } \\
\text { Atenafu, Eshetu; The Hospital for Sick Children, Child Health } \\
\text { Evaluative Sciences } \\
\text { Corey, Mary; Hospital for Sick Children } \\
\text { Ratjen, Felix; Hospital for Sick Children, Division of Resp. Medicine } \\
\text { Waters, Valerie; Hospital for Sick Children }\end{array}$ \\
\hline Keywords: & $\begin{array}{l}\text { methicillin resistant Staphylococcus aureus, mecA, pulsed-field gel } \\
\text { electrophoresis }\end{array}$ \\
\hline
\end{tabular}




\section{Epidemiology of borderline oxacillin-resistant Staphylococcus}

\section{aureus in pediatric cystic fibrosis.}

4 Timothy Ronan Leahy*, MB MRCPI, Institute of Molecular Medicine, Trinity College

5 Dublin, St James's Hospital, James's St, Dublin 8, Ireland; formerly of Division of

6 Infectious Diseases, Department of Pediatrics, The Hospital for Sick Children, Toronto.

7 Yvonne C.W. Yau, MD, FRCPC, Division of Microbiology, Department of Pediatric

8 Laboratory Medicine, The Hospital for Sick Children, Toronto.

9 Eshetu Atenafu, MSc, Child Evaluative Health Sciences, Research Institute, The Hospital

10 for Sick Children, Toronto.

11 Mary Corey, PhD, Child Evaluative Health Sciences, Research Institute, The Hospital for 12 Sick Children, Toronto.

13 Felix Ratjen, MD, PhD, FRCPC, Division of Respiratory Medicine, Department of

14 Pediatrics, The Hospital for Sick Children, Toronto.

15 Valerie Waters, MD, MSc, FRCPC Division of Infectious Diseases, Department of

16 Pediatrics, The Hospital for Sick Children, 555 University Avenue, Toronto, M5G 1X8,

17 Canada.

18 *Corresponding author: telephone number +35318961000 , fax number +3531

198963503 , email address leahyt@tcd.ie

20 Keywords: methicillin resistant Staphylococcus aureus (MRSA), mecA, pulsed-field gel

21 electrophoresis.

22 Abbreviated title. BORSA in pediatric cystic fibrosis 


\section{Summary}

25 A single-centre retrospective study was undertaken in children with cystic fibrosis (CF)

26 to evaluate 1) risk factors for acquisition; 2) molecular epidemiology; and 3) impact on

27 disease progression of borderline oxacillin-resistant Staphylococcus aureus (BORSA)

28 versus mecA-positive methicillin-resistant Staphylococcus aureus (MRSA). The study

29 comprised of 1) identification of all children with at least one respiratory specimen

30 positive for either BORSA or MRSA during the study period; 2) compilation of relevant

31 clinical and epidemiological data from 12-month period leading up to first positive

32 (index) culture; 3) microbiological and molecular characterisation of index isolates and 4)

33 measurement of subsequent clinical outcome. Thirty-eight children were identified with

34 at least one positive isolate; 4 were excluded due to insufficient clinical or laboratory

35 data. Eighteen children (53\%) grew BORSA in their index culture. Children who

36 acquired BORSA only $(\mathrm{n}=16)$ were more likely to have had prior MSSA colonization

$37(\mathrm{p}<0.0001)$. Usage of oral cephalexin $(\mathrm{p}<0.01)$ and inhaled tobramycin $(\mathrm{p}<0.03)$ prior to

38 index culture was significantly and independently associated with acquisition of BORSA.

39 The majority of BORSA isolates were hyper $\beta$-lactamase producers and susceptible to a

40 greater range of antibiotics. Strain relatedness was not evident within the BORSA group.

41 There was no difference in disease progression between the two groups. This is the first

42 study to demonstrate that a significant proportion of S. aureus isolates with methicillin

43 resistance in the CF population are BORSAs that lack mecA. Antibiotic pressure may

44 lead to the development of BORSA in CF patients. Prospective studies are needed to

45 assess its clinical impact. 


\section{Introduction}

49 respiratory pathogen in the cystic fibrosis $(\mathrm{CF})$ population. The US CF registry reported a

50 significant rise in MRSA infection among CF patients, from 2.1\% in 1996 to $21.2 \%$ in

$512007^{1}$. MRSA infection has been associated with accelerated deterioration in pulmonary

52 function ${ }^{2}$, increased hospitalization ${ }^{3}$, impaired growth, increased antibiotic usage ${ }^{4}$ and 53 increased mortality in CF patients ${ }^{5}$.

54 According to the Clinical Laboratory Standards Institute (CLSI), methicillin

55 resistance in S. aureus is defined as having an oxacillin minimum inhibitory

56 concentration (MIC) $>4 \mu \mathrm{g} / \mathrm{ml}^{6}$. The primary mechanism of resistance for MRSA is the

57 production of an altered penicillin binding protein (PBP2a) with reduced affinity for

58 methicillin that is encoded by the mecA gene ${ }^{7}$. We have identified a group of CF patients

59 infected with borderline resistant $S$. aureus (BORSA) isolates that are characterized by

60 oxacillin MICs at or just above the susceptibility breakpoint of $4 \mu \mathrm{g} / \mathrm{ml}$ and lack the

$61 m e c A$ gene $^{7}$. The implications of isolating BORSA in a respiratory culture from CF

62 patients have not previously been explored. The aim of this study was therefore to

63 compare the risk factors for acquisition, the molecular epidemiology and subsequent

64 clinical impact on disease progression of BORSA infection in comparison with mecA-

65 positive MRSA infection in pediatric CF patients.

66

67 


\section{Materials and Methods}

$70 \quad$ Study design and patient population

71 This was a single-centre retrospective comparative study of pediatric CF patients

72 whose care was primarily based at the CF clinic at the Hospital for Sick Children,

73 Toronto between 1992 and 2007. The clinic currently cares for approximately 280

74 children up to the age of eighteen. Respiratory samples (expectorated sputum or

75 oropharyngeal swabs) were sent from all children attending the clinic to the microbiology

76 laboratory for culture at every 3-month visit and during acute pulmonary exacerbations

77 (APEs). Patients with one or more respiratory specimens positive for S. aureus with

78 methicillin resistance (either BORSA or MRSA) were included in the analysis. Cases

79 with three or more specimens positive for BORSA or MRSA during the study period

80 were considered persistently colonized ${ }^{2}$. The study was approved by the Hospital for

81 Sick Children's Research Ethics Board.

82

83 Data collection

84 Patients with BORSA or MRSA isolated from their respiratory specimens were

85 identified using the microbiology database, and cross-referenced with the CF clinical

86 database. Date of the first BORSA or MRSA-positive respiratory culture (index culture)

87 for each patient was identified. The medical and laboratory records for each patient were

88 examined using a standardized data collection form. Baseline patient characteristics were

89 assessed at the date of index culture, and risk factors for acquisition of BORSA or MRSA

90 infection were assessed over the twelve-month period prior to the index culture. Impact 
91 on clinical progression was assessed by examination of the time period from index

92 culture until discharge from the clinic or until December $31^{\text {st }} 2008$, whichever date was

93 later. Data collected included information on patients' demographics, pulmonary function

94 tests (PFTs), respiratory cultures, hospital admissions, acute pulmonary exacerbations

95 and antibiotic use. An acute pulmonary exacerbation was defined as a change in

96 pulmonary status considered by the attending physician to require antibiotic treatment,

97 either oral or intravenous. An "antibiotic day" was defined as one day for each antibiotic

98 used, for example a 14-day course of two antibiotics was defined as 28 "antibiotic days".

99 The purpose of this distinction was to capture more complex antibiotic regimens that

100 might be employed to treat MRSA infected patients. Patients were classified as being on

101 oral cephalexin if they were prescribed cephalexin at the time of index culture of at least

102 one month's duration (in all instances, this occurred in the absence of signs or symptoms

103 of a pulmonary exacerbation). Patients were classified as being on inhaled tobramycin if

104 they were prescribed inhaled tobramycin at the time of index culture of at least one

105 month's duration (for previous $P$. aeruginosa infection). Patients were classified as being

106 on oral ciprofloxacin if they were prescribed at least one course of ciprofloxacin (in all

107 instances, this occurred in the presence of signs or symptoms of a pulmonary

108 exacerbation). Patients were categorized as co-colonized with other $\mathrm{CF}$ pathogens using

109 previously defined criteria ${ }^{8}$. Only patients with at least 6 months of follow-up after the

110 index culture were included in the analysis of outcome measures.

111

112

113 


\section{Microbiology}

\section{Specimen Processing}

116 Respiratory specimens from CF patients were screened for MRSA using media

117 containing $2 \mu \mathrm{g} / \mathrm{ml}$ of oxacillin: an in-house mannitol salt agar (MRSA agar base,

118 Acumedia, Lansing, MI, and 1\% D-mannitol, Difco, Detroit, MI ) supplemented with 1\%

119 thymidine and $2 \mu \mathrm{g} / \mathrm{ml}$ of oxacillin (both from Sigma, St. Louis, MO) was used from

1201992 to 2001, and Oxacillin Resistance Screening Agar Base (ORSAB, Oxoid, Nepean,

121 Ontario, Canada) was used from 2002 onward. Plates were incubated at $35^{\circ} \mathrm{C}$ aerobically

122 and observed for the presence of growth at 24 hours and at 48 hours. Suspicious colonies

123 were identified as $S$. aureus by Gram stain, catalase, slide coagulase, and tube coagulase

124 tests.

125

126 Antimicrobial Susceptibility

127 Antimicrobial susceptibity of $S$. aureus isolates was tested by a combination of

128 oxacillin $6 \mu \mathrm{g} / \mathrm{ml}$ screen plate, disk diffusion ${ }^{6,9}$ and an automated system (Vitek, bio-

129 Merieux, St. Laurent, Quebec, from 1992 to Nov 2002, and the BD Automated Phoenix

130 System, BD Diagnostic Systems, Sparks, MD from Nov 2002 onward). Inducible

131 resistance to clindamycin was detected by disk diffusion using the D-zone test ${ }^{6,9}$. In

132 addition, each strain was examined for the presence of PBP2a by latex agglutination

133 (PBP2' test kit, Oxoid, Hants, UK). Organisms that were negative for PBP2a by latex

134 agglutination but grew on the oxacillin screen plate were sent to the reference laboratory

135 (Central Provincial Health Laboratory, Ontario) for minimum inhibitory concentration

136 (MIC) by agar dilution and polymerase chain reaction (PCR) for the detection of the 
$137 m e c A$ gene. An isolate was deemed as BORSA if the oxacillin MIC was $\geq 4 \mu \mathrm{g} / \mathrm{ml}$,

138 PBP2a was absent and the mecA PCR was negative ${ }^{7}$. An isolate was deemed as MRSA if

139 the oxacillin MIC was $\geq 4 \mathrm{ug} / \mathrm{ml}$, the latex agglutination for PBP2a was positive and/or the 140 mecA PCR was positive ${ }^{6}$

\section{$142 \quad \beta$-Lactamase Production}

143 All BORSAs were tested for the production of $\beta$-lactamase as a potential

144 mechanism of oxacillin resistance by disk diffusion with ampicillin $(10 \mu \mathrm{g})$ and

145 amoxicillin-clavulinic $(20 \mu \mathrm{g} / 10 \mu \mathrm{g})$ disks as previously described (13). An isolate was

146 deemed as hyper $\beta$-lactamase producing if the ampicillin zone size was resistant and the

147 amoxicillin-clavulanic zone size was susceptible, suggesting borderline resistance was

148 secondary to large amounts of $\beta$ - actamase $^{9}(13)$.

150 Molecular Characterization

151 The mecA and nuc gene were co-amplified with a multiplex real time PCR as

152 described previously ${ }^{10}$. The presence of Panton-Valentine leukocidin was examined by

153 amplification of the $l u k F-P V$ and $l u k S-P V$ genes using primer and protocols previously

154 described ${ }^{11}$. Pulsed-field gel electrophoresis (PFGE) was conducted using SmaI as per

155 manufacturer protocol (Bio-rad Laboratories, Hercules, CA) on all newly identified

156 MRSA strains. Electrophoretically-generated DNA profiles were analyzed using the

157 criteria proposed by Tenover et al to determine strain relatedness ${ }^{12}$.

158

159 


\section{$160 \quad$ Statistical analysis}

161 Descriptive statistics, frequency distributions and percentages were calculated for

162 the outcome variables and other covariates of interest. Normality assumption test was

163 carried out for the continuous variables using the Kolmogorov-Smirnov test. Student's t-

164 test or Kruskal-Wallis test (if normality assumption failed) was conducted for continuous

165 variables within the two groups. The Chi-square or Fisher's exact test, as appropriate,

166 was carried out for the dichotomous variables to assess the association with the two

167 groups. To analyze independent risk factors, a multivariate analysis using a logistic

168 regression model was applied. A p-value of $<0.05$ was considered statistically significant.

169 The statistical programme SAS (version 9.1; SAS Institute Inc., Cary, NC) was used.

170 


\section{Results}

172

173 Patient population

174 Between 1992 and 2007, 38 patients had at least one respiratory specimen

175 positive for either BORSA or MRSA. Of these, three were excluded from our analysis

176 because their care was not primarily based at our hospital, thus we had no clinical data

177 for these patients. In addition, mecA PCR could not be performed on one isolate

178 retrospectively therefore 34 patients were included in the study. Of these 34 patients, two

179 patients were initially colonized with mecA-negative and subsequently mecA-positive

180 strains and were excluded from the final statistical analysis as it was unclear how to

181 categorize them in terms of patient characteristics and outcomes. Ultimately 16 patients

182 with mecA positive MRSA and 16 with $m e c A$ negative BORSA on the index culture were

183 compared for baseline characteristics. For the longitudinal assessment, 15 patients in each

184 group were compared since one patient in each group did not have follow-up data for at 185 least 6 months.

\section{Patient characteristics and risk factors for BORSA and MRSA}

188 The two groups were similar in terms of baseline characteristics (Table 1). There

189 was no difference in pulmonary function or rate of decline of pulmonary function in the

190 year prior to the index culture. In addition, there was no difference in the number of acute

191 pulmonary exacerbations, hospital admissions or overall antibiotic use for the treatment

192 of acute pulmonary exacerbations. A significantly greater proportion of BORSA infected

193 patients were colonized with MSSA in the year prior (94\% vs 25\%, p<0.0001). Likewise, 
194 a higher proportion of BORSA infected patients received oral antibiotics (cephalexin)

$195(75 \%$ vs $25 \%, \mathrm{p}=0.005)$.

196 Mean duration of therapy among those patients on cephalexin was 337 days (range 30-

197365 days) in the BORSA group and 311 days (range 150-365 days) in the MRSA group;

198 cephalexin treatment was instituted not for pulmonary exacerbation but as treatment for

199 MSSA identified on routine sputum cultures. Furthermore, a significantly greater

200 number of BORSA infected patients had received a course of oral ciprofloxacin (31\% vs

$2010 \%, \mathrm{p}=0.043)$ or were on inhaled antibiotics (tobramycin) $(88 \%$ vs $44 \%, \mathrm{p}=0.009)$ in the

202 year prior to the index culture than patients with MRSA. No inter-group difference was

203 detected in any of the other co-colonizing respiratory pathogens. Patients with BORSA

204 strains had significantly more clinic visits in the year prior to first isolate $(n=7.94)$ than

205 patients with mecA-positive strains $(\mathrm{n}=4.69, \mathrm{p}=0.049)$ although this was not a significant

206 risk factor in the logistic regression model

207 A logistic regression model was applied to determine the independence of

208 epidemiological and clinical variables that were correlated with BORSA infection.

209 Colonization with MSSA and use of oral cephalexin were significantly associated as $68 \%$

210 of patients with MSSA were treated with cephalexin and $81 \%$ of patients treated with

211 cephalexin were colonized with MSSA $(\mathrm{p}<0.05)$. The use of oral cephalexin (regression

212 coefficient $(\beta)=1.15, \mathrm{SD}=0.47, \mathrm{p}$ value $=0.015)$ and inhaled tobramycin (regression

213 coefficient $(\beta)=1.16, \mathrm{SD}=0.53$, $\mathrm{p}$ value $=0.028)$ in the year prior to index culture were

214 significantly and independently associated with BORSA infection.

215

216 Microbiological characteristics of BORSA and MRSA isolates 
217 The microbiological characteristics of the BORSA isolates are illustrated in Table

218 2. Fourteen of the 16 isolates had oxacillin MICs at or just above the breakpoint (4 to 8

$219 \mu \mathrm{g} / \mathrm{ml}$ ) but 2 isolates had MICs of $16 \mu \mathrm{g} / \mathrm{ml}$. All of the BORSA isolates were negative for 220 PBP2a by latex agglutination and were mecA negative by PCR. Twelve of the 16 BORSA

221 isolates were hyper $\beta$-lactamase producers. This was shown by resistance to ampicillin by

222 disk diffusion that was reverted to susceptible with the addition of clavulanic acid, a $\beta$ -

223 lactamase inhibitor. The remaining 4 isolates had ampicillin zone size that were

224 unchanged by addition of clavulin by disk diffusion.

225 In comparison to the MRSA isolates, a significantly greater proportion of the

226 BORSA isolates were susceptible to both erythromycin $(81 \%$ vs $13 \%, \mathrm{p}<0.0001)$ and

227 clindamycin $(94 \%$ vs $31 \%$, p<0.001). None of the BORSA isolates were D-test positive

228 but $5(45 \%)$ of the MRSA isolates were D-test positive $(\mathrm{p}=0.043)$. All of the BORSA

229 and MRSA isolates were susceptible to trimethoprim-sulphamethoxazole. None of the

230 BORSA isolates tested produced PVL. Three of the 16 MRSA isolates were identified as

231 USA300 epitypes, and produced PVL.

232

233 Relatedness of BORSA and MRSA isolates

234 Pulsed field gel electrophoresis (PFGE) revealed that none of the BORSA strains

235 were related by the Tenover criteria ${ }^{13}$ (Figure 1). Within the MRSA group, we found

236 four separate instances of strain relatedness during the course of the study: a pair of

237 siblings shared an indistinguishable strain, four patients shared possibly related strains,

238 two patients shared a closely related strain with a third patient sharing a possibly related 
239 strain and, finally, three patients (including a pair of siblings) shared an indistinguishable

240 strain.

241

242 Clinical outcomes of patients

243 To compare the clinical impact of BORSA versus MRSA infection on disease

244 progression, we assessed 99.7 patient-years of follow-up after the index culture with a

245 comparable mean duration of follow up of 3.37 years in the MRSA group and 3.27 years

246 in the BORSA group ( $\mathrm{p}=0.507)$ (Table 3). Patients infected with MRSA were more

247 likely to have persistent infection at the end of follow up than patients with BORSA

248 infection $(80 \%$ vs $53 \%)$ but this was not statistically significant $(\mathrm{p}=0.121)$. The annual

249 decline in percent predicted forced expiratory volume in 1 second $\left(\mathrm{FEV}_{1}\right)$, was greater

250 among the MRSA infected patients (5.8\%) than those infected with BORSA (2.8\%)

251 although this difference was not significant $(\mathrm{p}=0.77)$. Neither was there a significant

252 difference in the number of acute pulmonary exacerbations, hospital admissions, inpatient

253 days or clinic visits. One patient in the MRSA group died, and another progressed to lung

254 transplant. Three patients in the BORSA group had a lung transplant during the study

255 period.

256

John Wiley \& Sons, Inc. 


\section{Discussion}

258

259

260 isolates with methicillin resistance infecting CF patients are BORSA which lack the

261 mecA gene, the main genetic determinant of methicillin resistance in S. aureus ${ }^{7}$. The

262 present study demonstrates that antibiotic use in CF patients colonized with MSSA is a

263 risk factor specifically for infection with BORSA. Previous studies have shown that

264 ciprofloxacin and cephalosporin use is a risk factor for acquisition of MRSA in CF

265 patients ${ }^{14}$ but, to our knowledge, no other study has examined the differential risk factors

266 for BORSA compared to MRSA acquisition.

267

268 and even within households ${ }^{18}$ has been well described in the CF population. Evidence of

269 person to person transmission of $m e c A$-positive MRSA was also present in this study

270 with shared strains among CF patients and siblings. The mechanism of resistance is

271 relevant to how $\mathrm{CF}$ patients acquire MRSA as the mecA gene is absent in its entirety from

$272 \mathrm{MSSA}^{19} 7$. It is believed to have originated from another staphylococcus species, such as

273 Staphylococcus sciuri, and been transposed into MSSA ${ }^{20}$. Although horizontal transfer

274 of mecA between a S. epidermidis and MSSA isolate within the same patient has been

275 described $^{21}$, transmission of $m e c A$-positive MRSA isolates between individuals is likely

276 more common as there are a well defined number of mecA-positive MRSA clones

277 worldwide ${ }^{22}$.

278 Our data would suggest that $\mathrm{CF}$ patients may acquire mecA-negative BORSA

279 isolates in a different way. In contrast to MRSA isolates, BORSA isolates had unique 
280 PFGE patterns and appeared not to be shared between patients in our study. CF patients

281 colonized with MSSA and exposed to oral cephalexin or inhaled tobramycin (which can

282 act synergistically with $\beta$-lactam antibiotics against $S$. aureus) were at significantly

283 increased risk of acquiring BORSA infection. This suggests that BORSA isolates may

284 arise from endogenous MSSA isolates that develop other mechanisms of resistance to

285 methicillin in response to antibiotic pressure. Previous studies have shown that S. aureus

286 strains with borderline susceptibility to oxacillin have a significant drop in the penicillin

287 MICs to the susceptible range with the addition of a $\beta$-lactamase inhibitor ${ }^{23}$. This

288 predicts the response of oxacillin MICs to the addition of a $\beta$-lactamase inhibitor and

289 suggests that a large amount of $\beta$-lactamase production is the mechanism of resistance to

290 oxacillin in the majority of BORSA, as observed in 12 of 16 isolates in our study.

291 However, in the 4 remaining BORSA strains where the ampicillin zone size did not

292 change significantly with the addition of clavulanic acid, the mechanism of methicillin

293 resistance is unknown. In the aforementioned study, the authors described S. aureus

294 strains with intermediate MICs to oxacillin that remained the same in the presence of

295 clavulanic acid. The authors suggested that oxacillin resistance was due to factors other

296 than $\beta$-lactamase inactivation ${ }^{23}$. It is possible that it is due to a modification of "normal"

297 penicillin-binding proteins (PBPs) such as PBP 1,PBP 2 and PBP 4 as was previously

298 shown in clinical BORSA isolates by Tomasz et $\mathrm{al}^{24}$. Exposure of susceptible

299 staphylococci to increasing concentrations of $\beta$-lactam antibiotics in the laboratory is

300 known to cause PBP alterations associated with increased $\mathrm{MICs}^{25}$. In this study,

301 persistent antibiotic pressure may have similarly modified PBPs in endogenous MSSA

302 isolates colonizing CF patients and resulting in the development of mecA-negative, non- 
303 hyper $\beta$ lactamase producing BORSA isolates. Thus, in the prevention of the emergence

304 of BORSA in CF patients, other infection control measures, such as patient segregation, 305 may not be as important as the judicious use of antibiotics.

306 In our study, the majority of patients treated with oral cephalexin were colonized

307 with MSSA. Although there were no signs and symptoms of a pulmonary exacerbation at

308 the time, we are limited in a retrospective study to the information that is recorded in the

309 medical charts. Their antibiotic treatment was thus likely driven by their MSSA positive

310 respiratory cultures and/or more subtle symptoms. Primary prophylaxis for S. aureus (the

311 treatment of all newly diagnosed CF patients with antistaphylococcal agents irrespective

312 of respiratory culture results) is not routinely practiced in North America due to concerns

313 of $P$. aeruginosa acquisition ${ }^{26,27}$. A systematic review of prophylactic antibiotics in

314 cystic fibrosis, however, suggests that $P$. aeruginosa acquisition in this context is

315 associated less frequently with narrow spectrum anti-staphylococcal antibiotics such as

316 flucloxacillin compared with broader spectrum anti-staphylococcal antibiotics such as

317 cephalexin $^{28}$. In our study, although patients treated with oral cephalexin were not

318 receiving prophylaxis against $S$. aureus, they were receiving prolonged courses of

319 cephalexin for MSSA detected on routine culture of the respiratory tract. This prolonged

320 cephalexin use was identified as an independent risk factor for the acquisition of BORSA

321 isolates and occurred recently, primarily in the last 5 years of the study.

322 The clinical significance of BORSA infection in CF patients is unknown. Our

323 study lacked a sufficient sample size and is not powered to clearly delineate the clinical

324 impact of BORSA infection. Previous studies have reported accelerated deterioration in

325 pulmonary function ${ }^{2}$, increased hospitalization ${ }^{3}$, impaired growth, increased antibiotic 
326 usage ${ }^{4}$ and increased mortality ${ }^{5}$ in CF patients infected with MRSA. However, these

327 studies are limited by a lack of information on the laboratory methodology of identifying

328 MRSA and thus it is not known to what degree these effects are attributable to BORSA or

329 MRSA.

330 Detection of BORSA isolates may also influence the choice of antibiotics in the

331 treatment of these infections in the setting of a pulmonary exacerbation. In our study,

332 BORSA isolates were more susceptible to erythromycin and clindamycin than the MRSA

333 isolates. This finding is not surprising as the mecA gene is located on a mobile genetic

334 element (the Staphylococcal Cassette Chromosome mec: SCCmec) which can contain

335 additional drug resistance genes ${ }^{22}$. Although earlier studies suggested that infections due

336 to mecA negative BORSA isolates could be treated with $\beta$-lactam antibiotics ${ }^{29,30}$, more

337 recent cases report failure of cloxacillin and even vancomycin in the treatment of

338 endocarditis caused by BORSA, depending on the mechanism of methicillin resistance

33931,32 . This has implications for how respiratory specimens from CF patients should be

340 screened for MRSA. Some of the newer MRSA selective media such as chromogenic

341 media for MRSA that use cefoxitin as the selective agent will not detect mecA-negative

342 BORSA isolates as we have observed in our own clinical laboratory. Thus these isolates

343 could be missed from CF respiratory specimens unless full antimicrobial susceptibilities

344 were performed on all $S$. aureus isolates. Hence, CF patients could potentially be treated

345 with suboptimal antibiotics during a pulmonary exacerbation.

346 There were several limitations to this study. It is unclear whether CF patients

347 infected with BORSA isolates behave more like CF patients infected with MSSA or CF

348 patients infected with MRSA Future studies assessing the clinical impact of BORSA in 
349 CF should compare BORSA, MRSA and MSSA colonized patients in a longitudinal

350 fashion, controlling for potential confounding factors and include more extensive PFGE

351 comparisons to determine the evolution of BORSA. In addition, the small number of

352 patients limited the number of variables that could be included in the logistic regression

353 model of risk factors. This study was also not powered to detect differences in clinical

354 outcomes between the groups. It was a single centre study and may not reflect findings in

355 other CF populations. Finally, in a quarter of patients with mecA-negative BORSA, the

356 mechanism of resistance to methicillin remains unknown.

357 In conclusion, CF patients with mecA-positive MRSA most commonly acquire

358 their infection through person to person transmission whereas BORSA is likely

359 preferentially selected out from endogenous MSSA in CF patients due to persistent

360 antibiotic pressure. Prospective studies are needed to examine the prevalence, clinical

361 impact and appropriate management of BORSA in the CF population, as well as the

362 underlying mechanism of resistance.

363

364

365

366

367

John Wiley \& Sons, Inc. 


\section{Acknowledgements}

369 The authors wish to acknowledge the assistance of Dr ML Mulvey at the National

370 Reference Laboratory for his assistance in performing multiplex PCR for the MRSA

371 isolates, Dana Kovach, Division of Microbiology in performing the pulsed field gel

372 electrophoresis and Derek Stephens of the Child Health Evaluative Service for his

373 assistance in the data analysis. 


\section{References}

1. Cystic. Patient Registry 2007 Annual Report. Cystic Fibrosis Foundation.

2. Dasenbrook EC, Merlo CA, Diener-West M, Lechtzin N, Boyle MP. Persistent methicillin-resistant Staphylococcus aureus and rate of FEV1 decline in cystic fibrosis. Am J Respir Crit Care Med 2008;178(8):814-821.

3. Ren CL, Morgan WJ, Konstan MW, Schechter MS, Wagener JS, Fisher KA, Regelmann WE. Presence of methicillin resistant Staphylococcus aureus in respiratory cultures from cystic fibrosis patients is associated with lower lung function. Pediatr Pulmonol 2007;42(6):513-518.

4. Miall LS, McGinley NT, Brownlee KG, Conway SP. Methicillin resistant Staphylococcus aureus (MRSA) infection in cystic fibrosis. Arch Dis Child 2001;84(2):160-162.

5. Dasenbrook E, Checkley, W, Merlo, CA, Lechtzin, N, Boyle, MP. MRSA is associated with increased mortality: a 10 year cohort study of National Patient Registry. Pediatr Pulmonol 2009;Suppl. 32:310-311.

6. Clinical. Performance standards for antimicrobial susceptibility testing. Wayne, Pa: Clinical Laboratory Standards Institute; 2007.

7. Chambers HF. Methicillin resistance in staphylococci: molecular and biochemical basis and clinical implications. Clin Microbiol Rev 1997;10(4):781-791.

8. Lee TW, Brownlee KG, Conway SP, Denton M, Littlewood JM. Evaluation of a new definition for chronic Pseudomonas aeruginosa infection in cystic fibrosis patients. J Cyst Fibros 2003;2(1):29-34.

9. Clinical. performance standards for antimicrobial disk susceptiblity tests. Wayne, Pa: Clinical Laboratory Standards Institute; 2006.

10. Mulvey MR, MacDougall L, Cholin B, Horsman G, Fidyk M, Woods S. Community-associated methicillin-resistant Staphylococcus aureus, Canada. Emerg Infect Dis 2005;11(6):844-850.

11. Lina G, Piemont Y, Godail-Gamot F, Bes M, Peter MO, Gauduchon V, Vandenesch F, Etienne J. Involvement of Panton-Valentine leukocidin-producing Staphylococcus aureus in primary skin infections and pneumonia. Clin Infect Dis 1999;29(5):1128-1132.

12. Tenover FC, Arbeit RD, Goering RV, Mickelsen PA, Murray BE, Persing DH, Swaminathan B. Interpreting chromosomal DNA restriction patterns produced by pulsed-field gel electrophoresis: criteria for bacterial strain typing. J Clin Microbiol 1995;33(9):2233-2239.

13. Tenover F, Arbeit R, Goering R, Mickelsen P, Murray B, Persing D, Swaminathan B. Interpreting Chromosomal DNA Restriction Patterns Produced by Pulsed-Field Gel Electrophoresis: Criteria for Bacterial Strain Typing. Journal of Clinical Microbiology 1995;33(9):2233-2239.

14. Nadesalingam K, Conway SP, Denton M. Risk factors for acquisition of methicillin-resistant Staphylococcus aureus (MRSA) by patients with cystic fibrosis. J Cyst Fibros 2005;4(1):49-52.

15. Schlichting C, Branger C, Fournier JM, Witte W, Boutonnier A, Wolz C, Goullet $\mathrm{P}$, Doring G. Typing of Staphylococcus aureus by pulsed-field gel 
electrophoresis, zymotyping, capsular typing, and phage typing: resolution of clonal relationships. J Clin Microbiol 1993;31(2):227-232.

16. Givney R, Vickery A, Holliday A, Pegler M, Benn R. Methicillin-resistant Staphylococcus aureus in a cystic fibrosis unit. J Hosp Infect 1997;35(1):27-36.

17. Goodrich JS, Sutton-Shields TN, Kerr A, Wedd JP, Miller MB, Gilligan PH. Prevalence of community-associated methicillin-resistant Staphylococcus aureus in patients with cystic fibrosis. J Clin Microbiol 2009;47(4):1231-1233.

18. Stone A, Quittell L, Zhou J, Alba L, Bhat M, Decelie-Germana J, Rajan S, Bonitz L, Welter JJ, Dozor AJ, Gherson I, Lowy FD, Saiman L. Staphylococcus aureus Nasal Colonization Among Pediatric CF Patients and Their Household Contacts. Pediatr Infect Dis J 2009.

19. Kreiswirth B, Kornblum J, Arbeit RD, Eisner W, Maslow JN, McGeer A, Low DE, Novick RP. Evidence for a clonal origin of methicillin resistance in Staphylococcus aureus. Science 1993;259(5092):227-230.

20. Wu S, Piscitelli C, de Lencastre H, Tomasz A. Tracking the evolutionary origin of the methicillin resistance gene: cloning and sequencing of a homologue of mecA from a methicillin susceptible strain of Staphylococcus sciuri. Microb Drug Resist 1996;2(4):435-441.

21. Wielders CL, Vriens MR, Brisse S, de Graaf-Miltenburg LA, Troelstra A, Fleer A, Schmitz FJ, Verhoef J, Fluit AC. In-vivo transfer of mecA DNA to Staphylococcus aureus [corrected]. Lancet 2001;357(9269):1674-1675.

22. Deurenberg RH, Vink C, Kalenic S, Friedrich AW, Bruggeman CA, Stobberingh EE. The molecular evolution of methicillin-resistant Staphylococcus aureus. Clin Microbiol Infect 2007;13(3):222-235.

23. McDougal LK, Thornsberry C. The role of beta-lactamase in staphylococcal resistance to penicillinase-resistant penicillins and cephalosporins. J Clin Microbiol 1986;23(5):832-839.

24. Tomasz A, Drugeon HB, de Lencastre HM, Jabes D, McDougall L, Bille J. New mechanism for methicillin resistance in Staphylococcus aureus: clinical isolates that lack the PBP 2a gene and contain normal penicillin-binding proteins with modified penicillin-binding capacity. Antimicrob Agents Chemother 1989;33(11):1869-1874.

25. Tonin E, Tomasz A. Beta-lactam-specific resistant mutants of Staphylococcus aureus. Antimicrob Agents Chemother 1986;30(4):577-583.

26. Stutman HR, Lieberman JM, Nussbaum E, Marks MI. Antibiotic prophylaxis in infants and young children with cystic fibrosis: a randomized controlled trial. J Pediatr 2002;140(3):299-305.

27. Ratjen F, Comes G, Paul K, Posselt HG, Wagner TO, Harms K. Effect of continuous antistaphylococcal therapy on the rate of $\mathrm{P}$. aeruginosa acquisition in patients with cystic fibrosis. Pediatr Pulmonol 2001;31(1):13-16.

28. Smyth A, Walters S. Prophylactic antibiotics for cystic fibrosis. Cochrane Database Syst Rev 2003(3):CD001912. DOI 10.1002/14651858.CD001912.

29. Chambers HF, Archer G, Matsuhashi M. Low-level methicillin resistance in strains of Staphylococcus aureus. Antimicrob Agents Chemother 1989;33(4):424428. 
30. Massanari RM, Pfaller MA, Wakefield DS, Hammons GT, McNutt LA, Woolson RF, Helms CM. Implications of acquired oxacillin resistance in the management and control of Staphylococcus aureus infections. J Infect Dis 1988;158(4):702709.

31. Skinner S, Murray M, Walus T, Karlowsky JA. Failure of cloxacillin in treatment of a patient with borderline oxacillin-resistant Staphylococcus aureus endocarditis. J Clin Microbiol 2009;47(3):859-861.

32. Nelson L, Cockram CS, Lui G, Lam R, Lam E, Lai R, Ip M. Community case of methicillin-resistant Staphylococcus aureus infection. Emerg Infect Dis 2006;12(1):172-174. 


\section{Figure legends}

\section{Figure 1.}

A. PFGE of SmaI restriction fragments of representative MRSA isolates (lanes 2-6).

Strains in lanes 3, 4 are indistinguishable and strains in lanes 2, 6 are closely related.

Lanes 1,7: lambda ladder.

B. Representative BORSA isolates (lanes 1-5) that are unrelated. Lane 6: lambda ladder. 
1 Epidemiology of borderline oxacillin-resistant Staphylococcus

\section{aureus in pediatric cystic fibrosis.}

4 Timothy Ronan Leahy*, MB MRCPI, Institute of Molecular Medicine, Trinity College

5 Dublin, St James's Hospital, James's St, Dublin 8, Ireland; formerly of Division of

6 Infectious Diseases, Department of Pediatrics, The Hospital for Sick Children, Toronto.

7 Yvonne C.W. Yau, MD, FRCPC, Division of Microbiology, Department of Pediatric

8 Laboratory Medicine, The Hospital for Sick Children, Toronto.

9 Eshetu Atenafu, MSc, Child Evaluative Health Sciences, Research Institute, The Hospital

10 for Sick Children, Toronto.

11 Mary Corey, PhD, Child Evaluative Health Sciences, Research Institute, The Hospital for

12 Sick Children, Toronto.

13 Felix Ratjen, MD, PhD, FRCPC, Division of Respiratory Medicine, Department of

14 Pediatrics, The Hospital for Sick Children, Toronto.

15 Valerie Waters, MD, MSc, FRCPC Division of Infectious Diseases, Department of

16 Pediatrics, The Hospital for Sick Children, 555 University Avenue, Toronto, M5G 1X8,

17 Canada.

18 *Corresponding author: telephone number +35318961000 , fax number +3531

198963503 , email address leahyt@tcd.ie

20 Keywords: methicillin resistant Staphylococcus aureus (MRSA), mecA, pulsed-field gel

21 electrophoresis.

22 Abbreviated title. BORSA in pediatric cystic fibrosis 


\section{Summary}

25 A single-centre retrospective study was undertaken in children with cystic fibrosis (CF)

26 to evaluate 1) risk factors for acquisition; 2) molecular epidemiology; and 3) impact on

27 disease progression of borderline oxacillin-resistant Staphylococcus aureus (BORSA)

28 versus mecA-positive methicillin-resistant Staphylococcus aureus (MRSA). The study

29 comprised of 1) identification of all children with at least one respiratory specimen

30 positive for either BORSA or MRSA during the study period; 2) compilation of relevant

31 clinical and epidemiological data from 12-month period leading up to first positive

32 (index) culture; 3) microbiological and molecular characterisation of index isolates and 4)

33 measurement of subsequent clinical outcome. Thirty-eight children were identified with

34 at least one positive isolate; 4 were excluded due to insufficient clinical or laboratory

35 data. Eighteen children (53\%) grew BORSA in their index culture. Children who

36 acquired BORSA only $(\mathrm{n}=16)$ were more likely to have had prior MSSA colonization

$37(\mathrm{p}<0.0001)$. Usage of oral cephalexin $(\mathrm{p}<0.01)$ and inhaled tobramycin $(\mathrm{p}<0.03)$ prior to

38 index culture was significantly and independently associated with acquisition of BORSA.

39 The majority of BORSA isolates were hyper $\beta$-lactamase producers and susceptible to a

40 greater range of antibiotics. Strain relatedness was not evident within the BORSA group.

41 There was no difference in disease progression between the two groups. This is the first

42 study to demonstrate that a significant proportion of $S$. aureus isolates with methicillin

43 resistance in the CF population are BORSAs that lack mecA. Antibiotic pressure may

44 lead to the development of BORSA in CF patients. Prospective studies are needed to

45 assess its clinical impact. 


\section{Introduction}

47

49 respiratory pathogen in the cystic fibrosis $(\mathrm{CF})$ population. The US CF registry reported a

50 significant rise in MRSA infection among CF patients, from 2.1\% in 1996 to $21.2 \%$ in

$512007^{1}$. MRSA infection has been associated with accelerated deterioration in pulmonary

52 function ${ }^{2}$, increased hospitalization ${ }^{3}$, impaired growth, increased antibiotic usage ${ }^{4}$ and 53 increased mortality in CF patients ${ }^{5}$.

54 According to the Clinical Laboratory Standards Institute (CLSI), methicillin

55 resistance in $S$. aureus is defined as having an oxacillin minimum inhibitory

56 concentration (MIC) $>4 \mu \mathrm{g} / \mathrm{ml}^{6}$. The primary mechanism of resistance for MRSA is the

57 production of an altered penicillin binding protein (PBP2a) with reduced affinity for

58 methicillin that is encoded by the mecA gene ${ }^{7}$. We have identified a group of CF patients

59 infected with borderline resistant $S$. aureus (BORSA) isolates that are characterized by

60 oxacillin MICs at or just above the susceptibility breakpoint of $4 \mu \mathrm{g} / \mathrm{ml}$ and lack the

$61 m e c A$ gene $^{7}$. The implications of isolating BORSA in a respiratory culture from CF

62 patients have not previously been explored. The aim of this study was therefore to

63 compare the risk factors for acquisition, the molecular epidemiology and subsequent

64 clinical impact on disease progression of BORSA infection in comparison with mecA-

65 positive MRSA infection in pediatric CF patients. 


\section{Materials and Methods}

$70 \quad$ Study design and patient population

71 This was a single-centre retrospective comparative study of pediatric CF patients

72 whose care was primarily based at the CF clinic at the Hospital for Sick Children,

73 Toronto between 1992 and 2007. The clinic currently cares for approximately 280

74 children up to the age of eighteen. Respiratory samples (expectorated sputum or

75 oropharyngeal swabs) were sent from all children attending the clinic to the microbiology

76 laboratory for culture at every 3-month visit and during acute pulmonary exacerbations

77 (APEs). Patients with one or more respiratory specimens positive for S. aureus with

78 methicillin resistance (either BORSA or MRSA) were included in the analysis. Cases

79 with three or more specimens positive for BORSA or MRSA during the study period

80 were considered persistently colonized ${ }^{2}$. The study was approved by the Hospital for

81 Sick Children's Research Ethics Board.

82

83 Data collection

84 Patients with BORSA or MRSA isolated from their respiratory specimens were

85 identified using the microbiology database, and cross-referenced with the CF clinical

86 database. Date of the first BORSA or MRSA-positive respiratory culture (index culture)

87 for each patient was identified. The medical and laboratory records for each patient were

88 examined using a standardized data collection form. Baseline patient characteristics were

89 assessed at the date of index culture, and risk factors for acquisition of BORSA or MRSA

90 infection were assessed over the twelve-month period prior to the index culture. Impact 
91 on clinical progression was assessed by examination of the time period from index

92 culture until discharge from the clinic or until December $31^{\text {st }} 2008$, whichever date was

93 later. Data collected included information on patients' demographics, pulmonary function

94 tests (PFTs), respiratory cultures, hospital admissions, acute pulmonary exacerbations

95 and antibiotic use. An acute pulmonary exacerbation was defined as a change in

96 pulmonary status considered by the attending physician to require antibiotic treatment,

97 either oral or intravenous. An "antibiotic day" was defined as one day for each antibiotic

98 used, for example a 14-day course of two antibiotics was defined as 28 "antibiotic days".

99 The purpose of this distinction was to capture more complex antibiotic regimens that

100 might be employed to treat MRSA infected patients. Patients were classified as being on

101 oral cephalexin if they were prescribed cephalexin at the time of index culture of at least

102 one month's duration (in all instances, this occurred in the absence of signs or symptoms

103 of a pulmonary exacerbation). Patients were classified as being on inhaled tobramycin if

104 they were prescribed inhaled tobramycin at the time of index culture of at least one

105 month's duration (for previous P. aeruginosa infection). Patients were classified as being

106 on oral ciprofloxacin if they were prescribed at least one course of ciprofloxacin (in all

107 instances, this occurred in the presence of signs or symptoms of a pulmonary

108 exacerbation). Patients were categorized as co-colonized with other $\mathrm{CF}$ pathogens using

109 previously defined criteria ${ }^{8}$. Only patients with at least 6 months of follow-up after the

110 index culture were included in the analysis of outcome measures.

111

112

113 


\section{Microbiology}

\section{Specimen Processing}

116 Respiratory specimens from CF patients were screened for MRSA using media

117 containing $2 \mu \mathrm{g} / \mathrm{ml}$ of oxacillin: an in-house mannitol salt agar (MRSA agar base,

118 Acumedia, Lansing, MI, and 1\% D-mannitol, Difco, Detroit, MI ) supplemented with 1\%

119 thymidine and $2 \mu \mathrm{g} / \mathrm{ml}$ of oxacillin (both from Sigma, St. Louis, MO) was used from

1201992 to 2001, and Oxacillin Resistance Screening Agar Base (ORSAB, Oxoid, Nepean,

121 Ontario, Canada) was used from 2002 onward. Plates were incubated at $35^{\circ} \mathrm{C}$ aerobically

122 and observed for the presence of growth at 24 hours and at 48 hours. Suspicious colonies

123 were identified as $S$. aureus by Gram stain, catalase, slide coagulase, and tube coagulase

124 tests.

125

126 Antimicrobial Susceptibility

127 Antimicrobial susceptibity of $S$. aureus isolates was tested by a combination of

128 oxacillin $6 \mu \mathrm{g} / \mathrm{ml}$ screen plate, disk diffusion ${ }^{6,9}$ and an automated system (Vitek, bio-

129 Merieux, St. Laurent, Quebec, from 1992 to Nov 2002, and the BD Automated Phoenix

130 System, BD Diagnostic Systems, Sparks, MD from Nov 2002 onward). Inducible

131 resistance to clindamycin was detected by disk diffusion using the D-zone test ${ }^{6,9}$. In

132 addition, each strain was examined for the presence of PBP2a by latex agglutination

133 (PBP2' test kit, Oxoid, Hants, UK). Organisms that were negative for PBP2a by latex

134 agglutination but grew on the oxacillin screen plate were sent to the reference laboratory

135 (Central Provincial Health Laboratory, Ontario) for minimum inhibitory concentration

136 (MIC) by agar dilution and polymerase chain reaction (PCR) for the detection of the 
$137 m e c A$ gene. An isolate was deemed as BORSA if the oxacillin MIC was $\geq 4 \mu \mathrm{g} / \mathrm{ml}$,

138 PBP2a was absent and the mecA PCR was negative ${ }^{7}$. An isolate was deemed as MRSA if

139 the oxacillin MIC was $\geq 4 \mathrm{ug} / \mathrm{ml}$, the latex agglutination for PBP2a was positive and/or the 140 mecA PCR was positive ${ }^{6}$

\section{$142 \quad \beta$-Lactamase Production}

143 All BORSAs were tested for the production of $\beta$-lactamase as a potential

144 mechanism of oxacillin resistance by disk diffusion with ampicillin $(10 \mu \mathrm{g})$ and

145 amoxicillin-clavulinic $(20 \mu \mathrm{g} / 10 \mu \mathrm{g})$ disks as previously described (13). An isolate was

146 deemed as hyper $\beta$-lactamase producing if the ampicillin zone size was resistant and the

147 amoxicillin-clavulanic zone size was susceptible, suggesting borderline resistance was

148 secondary to large amounts of $\beta$ - lactamase $^{9}(13)$.

150 Molecular Characterization

151 The mecA and nuc gene were co-amplified with a multiplex real time PCR as

152 described previously ${ }^{10}$. The presence of Panton-Valentine leukocidin was examined by

153 amplification of the $l u k F-P V$ and $l u k S-P V$ genes using primer and protocols previously

154 described ${ }^{11}$. Pulsed-field gel electrophoresis (PFGE) was conducted using SmaI as per

155 manufacturer protocol (Bio-rad Laboratories, Hercules, CA) on all newly identified

156 MRSA strains. Electrophoretically-generated DNA profiles were analyzed using the

157 criteria proposed by Tenover et al to determine strain relatedness ${ }^{12}$. 


\section{$160 \quad$ Statistical analysis}

161 Descriptive statistics, frequency distributions and percentages were calculated for

162 the outcome variables and other covariates of interest. Normality assumption test was

163 carried out for the continuous variables using the Kolmogorov-Smirnov test. Student's t-

164 test or Kruskal-Wallis test (if normality assumption failed) was conducted for continuous

165 variables within the two groups. The Chi-square or Fisher's exact test, as appropriate,

166 was carried out for the dichotomous variables to assess the association with the two

167 groups. To analyze independent risk factors, a multivariate analysis using a logistic

168 regression model was applied. A p-value of $<0.05$ was considered statistically significant.

169 The statistical programme SAS (version 9.1; SAS Institute Inc., Cary, NC) was used.

170 


\section{Results}

172

173 Patient population

174 Between 1992 and 2007, 38 patients had at least one respiratory specimen

175 positive for either BORSA or MRSA. Of these, three were excluded from our analysis

176 because their care was not primarily based at our hospital, thus we had no clinical data

177 for these patients. In addition, mecA PCR could not be performed on one isolate

178 retrospectively therefore 34 patients were included in the study. Of these 34 patients, two

179 patients were initially colonized with mecA-negative and subsequently mecA-positive

180 strains and were excluded from the final statistical analysis as it was unclear how to

181 categorize them in terms of patient characteristics and outcomes. Ultimately 16 patients

182 with mecA positive MRSA and 16 with $m e c A$ negative BORSA on the index culture were

183 compared for baseline characteristics. For the longitudinal assessment, 15 patients in each

184 group were compared since one patient in each group did not have follow-up data for at 185 least 6 months.

\section{Patient characteristics and risk factors for BORSA and MRSA}

188 The two groups were similar in terms of baseline characteristics (Table 1). There

189 was no difference in pulmonary function or rate of decline of pulmonary function in the

190 year prior to the index culture. In addition, there was no difference in the number of acute

191 pulmonary exacerbations, hospital admissions or overall antibiotic use for the treatment

192 of acute pulmonary exacerbations. A significantly greater proportion of BORSA infected

193 patients were colonized with MSSA in the year prior (94\% vs 25\%, p<0.0001). Likewise, 
194 a higher proportion of BORSA infected patients received oral antibiotics (cephalexin)

$195(75 \%$ vs $25 \%, \mathrm{p}=0.005)$.

196 Mean duration of therapy among those patients on cephalexin was 337 days (range 30-

197365 days) in the BORSA group and 311 days (range 150-365 days) in the MRSA group;

198 cephalexin treatment was instituted not for pulmonary exacerbation but as treatment for

199 MSSA identified on routine sputum cultures. Furthermore, a significantly greater

200 number of BORSA infected patients had received a course of oral ciprofloxacin (31\% vs

$2010 \%, \mathrm{p}=0.043)$ or were on inhaled antibiotics (tobramycin) $(88 \%$ vs $44 \%, \mathrm{p}=0.009)$ in the

202 year prior to the index culture than patients with MRSA. No inter-group difference was

203 detected in any of the other co-colonizing respiratory pathogens. Patients with BORSA

204 strains had significantly more clinic visits in the year prior to first isolate $(n=7.94)$ than

205 patients with mecA-positive strains $(\mathrm{n}=4.69, \mathrm{p}=0.049)$ although this was not a significant

206 risk factor in the logistic regression model

207 A logistic regression model was applied to determine the independence of

208 epidemiological and clinical variables that were correlated with BORSA infection.

209 Colonization with MSSA and use of oral cephalexin were significantly associated as $68 \%$

210 of patients with MSSA were treated with cephalexin and $81 \%$ of patients treated with

211 cephalexin were colonized with MSSA $(\mathrm{p}<0.05)$. The use of oral cephalexin (regression

212 coefficient $(\beta)=1.15, \mathrm{SD}=0.47, \mathrm{p}$ value $=0.015)$ and inhaled tobramycin (regression

213 coefficient $(\beta)=1.16, \mathrm{SD}=0.53$, $\mathrm{p}$ value $=0.028)$ in the year prior to index culture were

214 significantly and independently associated with BORSA infection.

215

216 Microbiological characteristics of BORSA and MRSA isolates 
217 The microbiological characteristics of the BORSA isolates are illustrated in Table

218 2. Fourteen of the 16 isolates had oxacillin MICs at or just above the breakpoint (4 to 8

$219 \mu \mathrm{g} / \mathrm{ml}$ ) but 2 isolates had MICs of $16 \mu \mathrm{g} / \mathrm{ml}$. All of the BORSA isolates were negative for 220 PBP2a by latex agglutination and were mecA negative by PCR. Twelve of the 16 BORSA

221 isolates were hyper $\beta$-lactamase producers. This was shown by resistance to ampicillin by

222 disk diffusion that was reverted to susceptible with the addition of clavulanic acid, a $\beta$ -

223 lactamase inhibitor. The remaining 4 isolates had ampicillin zone size that were

224 unchanged by addition of clavulin by disk diffusion.

225 In comparison to the MRSA isolates, a significantly greater proportion of the

226 BORSA isolates were susceptible to both erythromycin $(81 \%$ vs $13 \%, \mathrm{p}<0.0001)$ and

227 clindamycin $(94 \%$ vs $31 \%, \mathrm{p}<0.001)$. None of the BORSA isolates were D-test positive

228 but $5(45 \%)$ of the MRSA isolates were D-test positive $(\mathrm{p}=0.043)$. All of the BORSA

229 and MRSA isolates were susceptible to trimethoprim-sulphamethoxazole. None of the

230 BORSA isolates tested produced PVL. Three of the 16 MRSA isolates were identified as

231 USA300 epitypes, and produced PVL.

232

233 Relatedness of BORSA and MRSA isolates

234 Pulsed field gel electrophoresis (PFGE) revealed that none of the BORSA strains

235 were related by the Tenover criteria ${ }^{13}$ (Figure 1). Within the MRSA group, we found

236 four separate instances of strain relatedness during the course of the study: a pair of

237 siblings shared an indistinguishable strain, four patients shared possibly related strains,

238 two patients shared a closely related strain with a third patient sharing a possibly related 
239 strain and, finally, three patients (including a pair of siblings) shared an indistinguishable

240 strain.

241

242 Clinical outcomes of patients

243 To compare the clinical impact of BORSA versus MRSA infection on disease

244 progression, we assessed 99.7 patient-years of follow-up after the index culture with a

245 comparable mean duration of follow up of 3.37 years in the MRSA group and 3.27 years

246 in the BORSA group ( $\mathrm{p}=0.507)$ (Table 3). Patients infected with MRSA were more

247 likely to have persistent infection at the end of follow up than patients with BORSA

248 infection $(80 \%$ vs $53 \%)$ but this was not statistically significant $(\mathrm{p}=0.121)$. The annual

249 decline in percent predicted forced expiratory volume in 1 second $\left(\mathrm{FEV}_{1}\right)$, was greater

250 among the MRSA infected patients (5.8\%) than those infected with BORSA (2.8\%)

251 although this difference was not significant $(\mathrm{p}=0.77)$. Neither was there a significant

252 difference in the number of acute pulmonary exacerbations, hospital admissions, inpatient

253 days or clinic visits. One patient in the MRSA group died, and another progressed to lung

254 transplant. Three patients in the BORSA group had a lung transplant during the study

255 period.

256

John Wiley \& Sons, Inc. 


\section{Discussion}

258

259

260 isolates with methicillin resistance infecting CF patients are BORSA which lack the

261 mecA gene, the main genetic determinant of methicillin resistance in S. aureus ${ }^{7}$. The

262 present study demonstrates that antibiotic use in CF patients colonized with MSSA is a

263 risk factor specifically for infection with BORSA. Previous studies have shown that

264 ciprofloxacin and cephalosporin use is a risk factor for acquisition of MRSA in CF

265 patients ${ }^{14}$ but, to our knowledge, no other study has examined the differential risk factors

266 for BORSA compared to MRSA acquisition.

267

268 and even within households ${ }^{18}$ has been well described in the CF population. Evidence of

269 person to person transmission of $m e c A$-positive MRSA was also present in this study

270 with shared strains among CF patients and siblings. The mechanism of resistance is

271 relevant to how $\mathrm{CF}$ patients acquire MRSA as the mecA gene is absent in its entirety from

$272 \mathrm{MSSA}^{19} 7$. It is believed to have originated from another staphylococcus species, such as

273 Staphylococcus sciuri, and been transposed into MSSA ${ }^{20}$. Although horizontal transfer

274 of mecA between a S. epidermidis and MSSA isolate within the same patient has been

275 described $^{21}$, transmission of $m e c A$-positive MRSA isolates between individuals is likely

276 more common as there are a well defined number of mecA-positive MRSA clones

277 worldwide ${ }^{22}$.

278 Our data would suggest that $\mathrm{CF}$ patients may acquire mecA-negative BORSA

279 isolates in a different way. In contrast to MRSA isolates, BORSA isolates had unique 
280 PFGE patterns and appeared not to be shared between patients in our study. CF patients

281 colonized with MSSA and exposed to oral cephalexin or inhaled tobramycin (which can

282 act synergistically with $\beta$-lactam antibiotics against $S$. aureus) were at significantly

283 increased risk of acquiring BORSA infection. This suggests that BORSA isolates may

284 arise from endogenous MSSA isolates that develop other mechanisms of resistance to

285 methicillin in response to antibiotic pressure. Previous studies have shown that S. aureus

286 strains with borderline susceptibility to oxacillin have a significant drop in the penicillin

287 MICs to the susceptible range with the addition of a $\beta$-lactamase inhibitor ${ }^{23}$. This

288 predicts the response of oxacillin MICs to the addition of a $\beta$-lactamase inhibitor and

289 suggests that a large amount of $\beta$-lactamase production is the mechanism of resistance to

290 oxacillin in the majority of BORSA, as observed in 12 of 16 isolates in our study.

291 However, in the 4 remaining BORSA strains where the ampicillin zone size did not

292 change significantly with the addition of clavulanic acid, the mechanism of methicillin

293 resistance is unknown. In the aforementioned study, the authors described S. aureus

294 strains with intermediate MICs to oxacillin that remained the same in the presence of

295 clavulanic acid. The authors suggested that oxacillin resistance was due to factors other

296 than $\beta$-lactamase inactivation ${ }^{23}$. It is possible that it is due to a modification of "normal"

297 penicillin-binding proteins (PBPs) such as PBP 1,PBP 2 and PBP 4 as was previously

298 shown in clinical BORSA isolates by Tomasz et $\mathrm{al}^{24}$. Exposure of susceptible

299 staphylococci to increasing concentrations of $\beta$-lactam antibiotics in the laboratory is

300 known to cause PBP alterations associated with increased $\mathrm{MICs}^{25}$. In this study,

301 persistent antibiotic pressure may have similarly modified PBPs in endogenous MSSA

302 isolates colonizing CF patients and resulting in the development of mecA-negative, non- 
303 hyper $\beta$ lactamase producing BORSA isolates. Thus, in the prevention of the emergence

304 of BORSA in CF patients, other infection control measures, such as patient segregation, 305 may not be as important as the judicious use of antibiotics.

306 In our study, the majority of patients treated with oral cephalexin were colonized

307 with MSSA. Although there were no signs and symptoms of a pulmonary exacerbation at

308 the time, we are limited in a retrospective study to the information that is recorded in the

309 medical charts. Their antibiotic treatment was thus likely driven by their MSSA positive

310 respiratory cultures and/or more subtle symptoms. Primary prophylaxis for $S$. aureus (the

311 treatment of all newly diagnosed CF patients with antistaphylococcal agents irrespective

312 of respiratory culture results) is not routinely practiced in North America due to concerns

313 of $P$. aeruginosa acquisition ${ }^{26,27}$. A systematic review of prophylactic antibiotics in

314 cystic fibrosis, however, suggests that $P$. aeruginosa acquisition in this context is

315 associated less frequently with narrow spectrum anti-staphylococcal antibiotics such as

316 flucloxacillin compared with broader spectrum anti-staphylococcal antibiotics such as

317 cephalexin $^{28}$. In our study, although patients treated with oral cephalexin were not

318 receiving prophylaxis against $S$. aureus, they were receiving prolonged courses of

319 cephalexin for MSSA detected on routine culture of the respiratory tract. This prolonged

320 cephalexin use was identified as an independent risk factor for the acquisition of BORSA

321 isolates and occurred recently, primarily in the last 5 years of the study.

322 The clinical significance of BORSA infection in CF patients is unknown. Our

323 study lacked a sufficient sample size and is not powered to clearly delineate the clinical

324 impact of BORSA infection. Previous studies have reported accelerated deterioration in

325 pulmonary function ${ }^{2}$, increased hospitalization ${ }^{3}$, impaired growth, increased antibiotic 
326 usage ${ }^{4}$ and increased mortality ${ }^{5}$ in CF patients infected with MRSA. However, these

327 studies are limited by a lack of information on the laboratory methodology of identifying

328 MRSA and thus it is not known to what degree these effects are attributable to BORSA or

329 MRSA.

330 Detection of BORSA isolates may also influence the choice of antibiotics in the

331 treatment of these infections in the setting of a pulmonary exacerbation. In our study,

332 BORSA isolates were more susceptible to erythromycin and clindamycin than the MRSA

333 isolates. This finding is not surprising as the mecA gene is located on a mobile genetic

334 element (the Staphylococcal Cassette Chromosome mec: SCCmec) which can contain

335 additional drug resistance genes ${ }^{22}$. Although earlier studies suggested that infections due

336 to mecA negative BORSA isolates could be treated with $\beta$-lactam antibiotics ${ }^{29,30}$, more

337 recent cases report failure of cloxacillin and even vancomycin in the treatment of

338 endocarditis caused by BORSA, depending on the mechanism of methicillin resistance

33931,32 . This has implications for how respiratory specimens from CF patients should be

340 screened for MRSA. Some of the newer MRSA selective media such as chromogenic

341 media for MRSA that use cefoxitin as the selective agent will not detect mecA-negative

342 BORSA isolates as we have observed in our own clinical laboratory. Thus these isolates

343 could be missed from CF respiratory specimens unless full antimicrobial susceptibilities

344 were performed on all $S$. aureus isolates. Hence, CF patients could potentially be treated

345 with suboptimal antibiotics during a pulmonary exacerbation.

346 There were several limitations to this study. It is unclear whether CF patients

347 infected with BORSA isolates behave more like CF patients infected with MSSA or CF

348 patients infected with MRSA Future studies assessing the clinical impact of BORSA in 
349 CF should compare BORSA, MRSA and MSSA colonized patients in a longitudinal

350 fashion, controlling for potential confounding factors and include more extensive PFGE

351 comparisons to determine the evolution of BORSA. In addition, the small number of

352 patients limited the number of variables that could be included in the logistic regression

353 model of risk factors. This study was also not powered to detect differences in clinical

354 outcomes between the groups. It was a single centre study and may not reflect findings in

355 other CF populations. Finally, in a quarter of patients with mecA-negative BORSA, the

356 mechanism of resistance to methicillin remains unknown.

357 In conclusion, CF patients with mecA-positive MRSA most commonly acquire

358 their infection through person to person transmission whereas BORSA is likely

359 preferentially selected out from endogenous MSSA in CF patients due to persistent

360 antibiotic pressure. Prospective studies are needed to examine the prevalence, clinical

361 impact and appropriate management of BORSA in the CF population, as well as the

362 underlying mechanism of resistance.

363

364

365

366

367

John Wiley \& Sons, Inc. 


\section{Acknowledgements}

369 The authors wish to acknowledge the assistance of Dr ML Mulvey at the National

370 Reference Laboratory for his assistance in performing multiplex PCR for the MRSA

371 isolates, Dana Kovach, Division of Microbiology in performing the pulsed field gel

372 electrophoresis and Derek Stephens of the Child Health Evaluative Service for his

373 assistance in the data analysis. 


\section{References}

1. Cystic. Patient Registry 2007 Annual Report. Cystic Fibrosis Foundation.

2. Dasenbrook EC, Merlo CA, Diener-West M, Lechtzin N, Boyle MP. Persistent methicillin-resistant Staphylococcus aureus and rate of FEV1 decline in cystic fibrosis. Am J Respir Crit Care Med 2008;178(8):814-821.

3. Ren CL, Morgan WJ, Konstan MW, Schechter MS, Wagener JS, Fisher KA, Regelmann WE. Presence of methicillin resistant Staphylococcus aureus in respiratory cultures from cystic fibrosis patients is associated with lower lung function. Pediatr Pulmonol 2007;42(6):513-518.

4. Miall LS, McGinley NT, Brownlee KG, Conway SP. Methicillin resistant Staphylococcus aureus (MRSA) infection in cystic fibrosis. Arch Dis Child 2001;84(2):160-162.

5. Dasenbrook E, Checkley, W, Merlo, CA, Lechtzin, N, Boyle, MP. MRSA is associated with increased mortality: a 10 year cohort study of National Patient Registry. Pediatr Pulmonol 2009;Suppl. 32:310-311.

6. Clinical. Performance standards for antimicrobial susceptibility testing. Wayne, Pa: Clinical Laboratory Standards Institute; 2007.

7. Chambers HF. Methicillin resistance in staphylococci: molecular and biochemical basis and clinical implications. Clin Microbiol Rev 1997;10(4):781-791.

8. Lee TW, Brownlee KG, Conway SP, Denton M, Littlewood JM. Evaluation of a new definition for chronic Pseudomonas aeruginosa infection in cystic fibrosis patients. J Cyst Fibros 2003;2(1):29-34.

9. Clinical. performance standards for antimicrobial disk susceptiblity tests. Wayne, Pa: Clinical Laboratory Standards Institute; 2006.

10. Mulvey MR, MacDougall L, Cholin B, Horsman G, Fidyk M, Woods S. Community-associated methicillin-resistant Staphylococcus aureus, Canada. Emerg Infect Dis 2005;11(6):844-850.

11. Lina G, Piemont Y, Godail-Gamot F, Bes M, Peter MO, Gauduchon V, Vandenesch F, Etienne J. Involvement of Panton-Valentine leukocidin-producing Staphylococcus aureus in primary skin infections and pneumonia. Clin Infect Dis 1999;29(5):1128-1132.

12. Tenover FC, Arbeit RD, Goering RV, Mickelsen PA, Murray BE, Persing DH, Swaminathan B. Interpreting chromosomal DNA restriction patterns produced by pulsed-field gel electrophoresis: criteria for bacterial strain typing. J Clin Microbiol 1995;33(9):2233-2239.

13. Tenover F, Arbeit R, Goering R, Mickelsen P, Murray B, Persing D, Swaminathan B. Interpreting Chromosomal DNA Restriction Patterns Produced by Pulsed-Field Gel Electrophoresis: Criteria for Bacterial Strain Typing. Journal of Clinical Microbiology 1995;33(9):2233-2239.

14. Nadesalingam K, Conway SP, Denton M. Risk factors for acquisition of methicillin-resistant Staphylococcus aureus (MRSA) by patients with cystic fibrosis. J Cyst Fibros 2005;4(1):49-52.

15. Schlichting C, Branger C, Fournier JM, Witte W, Boutonnier A, Wolz C, Goullet $\mathrm{P}$, Doring G. Typing of Staphylococcus aureus by pulsed-field gel 
electrophoresis, zymotyping, capsular typing, and phage typing: resolution of clonal relationships. J Clin Microbiol 1993;31(2):227-232.

16. Givney R, Vickery A, Holliday A, Pegler M, Benn R. Methicillin-resistant Staphylococcus aureus in a cystic fibrosis unit. J Hosp Infect 1997;35(1):27-36.

17. Goodrich JS, Sutton-Shields TN, Kerr A, Wedd JP, Miller MB, Gilligan PH. Prevalence of community-associated methicillin-resistant Staphylococcus aureus in patients with cystic fibrosis. J Clin Microbiol 2009;47(4):1231-1233.

18. Stone A, Quittell L, Zhou J, Alba L, Bhat M, Decelie-Germana J, Rajan S, Bonitz L, Welter JJ, Dozor AJ, Gherson I, Lowy FD, Saiman L. Staphylococcus aureus Nasal Colonization Among Pediatric CF Patients and Their Household Contacts. Pediatr Infect Dis J 2009.

19. Kreiswirth B, Kornblum J, Arbeit RD, Eisner W, Maslow JN, McGeer A, Low DE, Novick RP. Evidence for a clonal origin of methicillin resistance in Staphylococcus aureus. Science 1993;259(5092):227-230.

20. Wu S, Piscitelli C, de Lencastre H, Tomasz A. Tracking the evolutionary origin of the methicillin resistance gene: cloning and sequencing of a homologue of mecA from a methicillin susceptible strain of Staphylococcus sciuri. Microb Drug Resist 1996;2(4):435-441.

21. Wielders CL, Vriens MR, Brisse S, de Graaf-Miltenburg LA, Troelstra A, Fleer A, Schmitz FJ, Verhoef J, Fluit AC. In-vivo transfer of mecA DNA to Staphylococcus aureus [corrected]. Lancet 2001;357(9269):1674-1675.

22. Deurenberg RH, Vink C, Kalenic S, Friedrich AW, Bruggeman CA, Stobberingh EE. The molecular evolution of methicillin-resistant Staphylococcus aureus. Clin Microbiol Infect 2007;13(3):222-235.

23. McDougal LK, Thornsberry C. The role of beta-lactamase in staphylococcal resistance to penicillinase-resistant penicillins and cephalosporins. J Clin Microbiol 1986;23(5):832-839.

24. Tomasz A, Drugeon HB, de Lencastre HM, Jabes D, McDougall L, Bille J. New mechanism for methicillin resistance in Staphylococcus aureus: clinical isolates that lack the PBP 2a gene and contain normal penicillin-binding proteins with modified penicillin-binding capacity. Antimicrob Agents Chemother 1989;33(11):1869-1874.

25. Tonin E, Tomasz A. Beta-lactam-specific resistant mutants of Staphylococcus aureus. Antimicrob Agents Chemother 1986;30(4):577-583.

26. Stutman HR, Lieberman JM, Nussbaum E, Marks MI. Antibiotic prophylaxis in infants and young children with cystic fibrosis: a randomized controlled trial. J Pediatr 2002;140(3):299-305.

27. Ratjen F, Comes G, Paul K, Posselt HG, Wagner TO, Harms K. Effect of continuous antistaphylococcal therapy on the rate of $\mathrm{P}$. aeruginosa acquisition in patients with cystic fibrosis. Pediatr Pulmonol 2001;31(1):13-16.

28. Smyth A, Walters S. Prophylactic antibiotics for cystic fibrosis. Cochrane Database Syst Rev 2003(3):CD001912. DOI 10.1002/14651858.CD001912.

29. Chambers HF, Archer G, Matsuhashi M. Low-level methicillin resistance in strains of Staphylococcus aureus. Antimicrob Agents Chemother 1989;33(4):424428. 
30. Massanari RM, Pfaller MA, Wakefield DS, Hammons GT, McNutt LA, Woolson RF, Helms CM. Implications of acquired oxacillin resistance in the management and control of Staphylococcus aureus infections. J Infect Dis 1988;158(4):702709.

31. Skinner S, Murray M, Walus T, Karlowsky JA. Failure of cloxacillin in treatment of a patient with borderline oxacillin-resistant Staphylococcus aureus endocarditis. J Clin Microbiol 2009;47(3):859-861.

32. Nelson L, Cockram CS, Lui G, Lam R, Lam E, Lai R, Ip M. Community case of methicillin-resistant Staphylococcus aureus infection. Emerg Infect Dis 2006;12(1):172-174. 


\section{Figure legends}

\section{Figure 1.}

A. PFGE of SmaI restriction fragments of representative MRSA isolates (lanes 2-6).

Strains in lanes 3, 4 are indistinguishable and strains in lanes 2, 6 are closely related.

Lanes 1,7: lambda ladder.

B. Representative BORSA isolates (lanes 1-5) that are unrelated. Lane 6: lambda ladder. 
Table 1. Characteristics of patients with MRSA and BORSA

Mean age (range) in $\mathrm{yr}$

No. (\%) male

Mean BMI z-score

No. (\%) homozygous DF508

No. (\%) pancreatic insufficient

No. (\%) CF related diabetes

Mean $\%$ predicted $\mathrm{FEV}_{1}$ (range)

$10.4(1.8-17.5)$
$10(63)$
-0.18
$7(44)$
$15(94)$
$1(6)$

$68.5(41-99)$

$\begin{array}{cc}13.1(8.5-17.3) & 0.078 \\ 8(50) & 0.476 \\ -0.40 & >0.5 \\ 7(44) & >0.5 \\ 14(88) & >0.5 \\ 2(13) & >0.5 \\ 69.4(30-113) & >0.5\end{array}$

$4(25)$

$3(19)$

$1(6)$

$1(6)$

$1(6)$

$1(6)$

$15(94)$

$<0.0001$

$6(38)$

$5(31)$

$1(6)$

$0(0)$

$1(6)$

0.433

0.171

$>0.5$

$>0.5$

$>0.5$

Antibiotic usage in 12-month period prior to index culture

No. (\%) of patients on oral cephalexin

$4(25)$

$12(75)$

0.005

7 (44)

$14(88)$

0.009

$0(0)$

0.043

\section{Disease activity during 12-month period prior to index culture}

Mean change (range) in \% predicted $\mathrm{FEV}_{1}$

Mean no. (range) of exacerbations $(-11.6$ to +10.7$)$ $(-28.9$ to +10.2$)$

Mean no. (range) of hospital admissions

$0.8(0-4)$

$1.4(0-8)$

$>0.5$

Mean no. (range) of inpatient days

$0.4(0-4)$

$0.7(0-6)$

$>0.5$

Mean no. (range) of IV antibiotic days

$3.4(0-37)$

$7.6(0-61)$

$>0.5$

$6.1(0-70)$

$14.2(0-130)$

Mean no. (range) of clinic visits 0.049

Abbreviations: BMI - body mass index, MSSA- methicillin sensitive Staphylococcus aureus, $\mathrm{FEV}_{1^{-}}$ Forced expiratory volume in 1 second. 
Table 1. Characteristics of patients with MRSA and BORSA

\section{Patient characteristics at index culture}

Mean age (range) in yr

No. (\%) male

Mean BMI z-score

No. (\%) homozygous DF508

No. (\%) pancreatic insufficient

No. (\%) CF related diabetes

Mean $\%$ predicted $\mathrm{FEV}_{1}$ (range)

$10.4(1.8-17.5)$
$10(63)$
-0.18
$7(44)$
$15(94)$
$1(6)$

$68.5(41-99)$

$\begin{array}{cc}13.1(8.5-17.3) & 0.078 \\ 8(50) & 0.476 \\ -0.40 & >0.5 \\ 7(44) & >0.5 \\ 14(88) & >0.5 \\ 2(13) & >0.5 \\ 69.4(30-113) & >0.5\end{array}$

$15(94)$

$<0.0001$

$6(38)$

0.433

$5(31)$

0.171

$1(6)$

$1(6)$

$>0.5$

$0(0)$

$1(6)$

$>0.5$

$1(6)$

$1(6)$

$4(25)$

$12(75)$

$14(88)$

0.005

$7(44)$

$5(31)$

0.043

\section{Disease activity during 12-month period prior to index culture}

Mean change (range) in \% predicted $\mathrm{FEV}_{1}$

Mean no. (range) of exacerbations $(-11.6$ to +10.7$)$ $(-28.9$ to +10.2$)$

Mean no. (range) of hospital admissions

$0.8(0-4)$

$1.4(0-8)$

$>0.5$

Mean no. (range) of inpatient days

$0.4(0-4)$

$0.7(0-6)$

Mean no. (range) of IV antibiotic days

$6.1(0-70)$

$14.2(0-130)$

Mean no. (range) of clinic visits 0.049

Abbreviations: BMI - body mass index, MSSA- methicillin sensitive Staphylococcus aureus, $\mathrm{FEV}_{1^{-}}$ Forced expiratory volume in 1 second. 
Table 2. Microbiological characteristics of BORSA isolates

\begin{tabular}{|c|c|c|c|c|c|c|c|c|}
\hline Isolate & $\begin{array}{l}\text { Oxacillin MIC } \\
(\mu \mathrm{g} / \mathrm{ml})\end{array}$ & $\begin{array}{l}\text { PBP2a latex } \\
\text { agglutination }\end{array}$ & $\begin{array}{l}\text { mecA } \\
\text { PCR }\end{array}$ & $\begin{array}{l}\text { AMP/AMC } \\
\text { zone size } \\
(\mathrm{mm}) \dagger\end{array}$ & $\begin{array}{l}\text { Hyper } \beta- \\
\text { lactamase } \\
\text { production }\end{array}$ & ERY & CLI & SXT \\
\hline SA 002 & 8 & neg & neg & $13 / 23$ & Yes & $S$ & $\mathrm{~S}$ & $S$ \\
\hline SA 003 & 16 & neg & neg & $12 / 26$ & Yes & I & $\mathrm{S}^{*}$ & $\mathrm{~S}$ \\
\hline SA 004 & 4 & neg & neg & $38 / 40$ & No & $\mathrm{S}$ & $\mathrm{S}$ & $S$ \\
\hline SA 006 & 8 & neg & neg & $35 / 36$ & No & $\mathrm{S}$ & $\mathrm{S}$ & $S$ \\
\hline SA 011 & 8 & neg & neg & $18 / 29$ & Yes & $S$ & $\mathrm{~S}$ & $S$ \\
\hline SA 016 & 4 & neg & neg & $15 / 26$ & Yes & $\mathrm{S}$ & $\mathrm{S}$ & $S$ \\
\hline SA 018 & 8 & neg & neg & $14 / 28$ & Yes & $\mathrm{S}$ & $\mathrm{S}$ & $S$ \\
\hline SA 020 & 4 & neg & neg & $17 / 28$ & Yes & $\mathrm{S}$ & $\mathrm{S}$ & $\mathrm{S}$ \\
\hline SA 021 & 4 & neg & neg & $16 / 26$ & Yes & $\mathrm{S}$ & $\mathrm{S}$ & $S$ \\
\hline SA 022 & 8 & neg & neg & $30 / 29$ & No & $\mathrm{S}$ & $\mathrm{S}$ & $S$ \\
\hline SA 023 & 8 & neg & neg & $16 / 21$ & Yes & $\mathrm{R}$ & $S^{*}$ & $\mathrm{~S}$ \\
\hline SA 029 & 16 & neg & neg & $10 / 27$ & Yes & $\mathrm{S}$ & $\mathrm{S}$ & $S$ \\
\hline SA 034 & 8 & neg & neg & $12 / 21$ & Yes & $\mathrm{S}$ & $S$ & $S$ \\
\hline SA 035 & 4 & neg & neg & $29 / 31$ & No & $\mathrm{S}$ & $\mathrm{S}$ & $S$ \\
\hline SA 036 & 4 & neg & neg & $16 / 23$ & Yes & $\mathrm{S}$ & $S$ & $S$ \\
\hline SA 038 & 8 & neg & neg & $11 / 24$ & Yes & $\mathrm{R}$ & $\mathrm{S}^{*}$ & $\mathrm{~S}$ \\
\hline
\end{tabular}

$\dagger$ Ampicillin (AMP) susceptible zone size $\geq 29 \mathrm{~mm}$, resistant $\leq 28 \mathrm{~mm}$ Amoxicillin clavulanic acid (AMC) susceptible zone size $\geq 20 \mathrm{~mm}$, resistant $\leq 19 \mathrm{~mm}^{9}$ * D-test negative, AMP-ampicillin, AMC-amoxicillin clavulanic acid ERY-erythromycin, CLI-clindamycin, SXT-trimethoprim-sulphamethoxazole S-susceptible I-intermediate R-resistant 
Table 3. Clinical outcomes of patients with MRSA and BORSA

\begin{tabular}{lccc}
\hline & MRSA & BORSA & P- \\
\hline \multicolumn{1}{c}{ Clinical outcomes } & $(\mathbf{n}=\mathbf{1 5})$ & value \\
& & & \\
& & & \\
Mean duration (range) of follow up in years & $3.37(0.76-14.8)$ & $3.27(0.66-5.57)$ & 0.507 \\
Mean annual change (range) in \% predicted FEV1 & $-5.8(-33.1$ to +3.2) & $-2.8(-10.2$ to +7.7) & 0.770 \\
Mean no. (range) of exacerbations per year & $1.8(0-5.3)$ & $2.4(0.3-7.6)$ & 0.443 \\
Mean no. (range) of hospital admissions per year & $0.9(0-6.6)$ & $1.3(0-4.7)$ & 0.242 \\
Mean no. (range) of inpatient days per year & $11.5(0-109.2)$ & $16.1(0-91.7)$ & 0.226 \\
Mean no. (range) of IV antibiotic days per year & $26.1(0-224.1)$ & $33.3(0-200.6)$ & 0.361 \\
Mean no. (range) of clinic visits per year & $7.7(3.7-37.4)$ & $11.7(2.8-80.9)$ & 0.272 \\
No. (\%) of patients with persistent infection & $12(80)$ & $8(53.3)$ & 0.121 \\
\hline
\end{tabular}




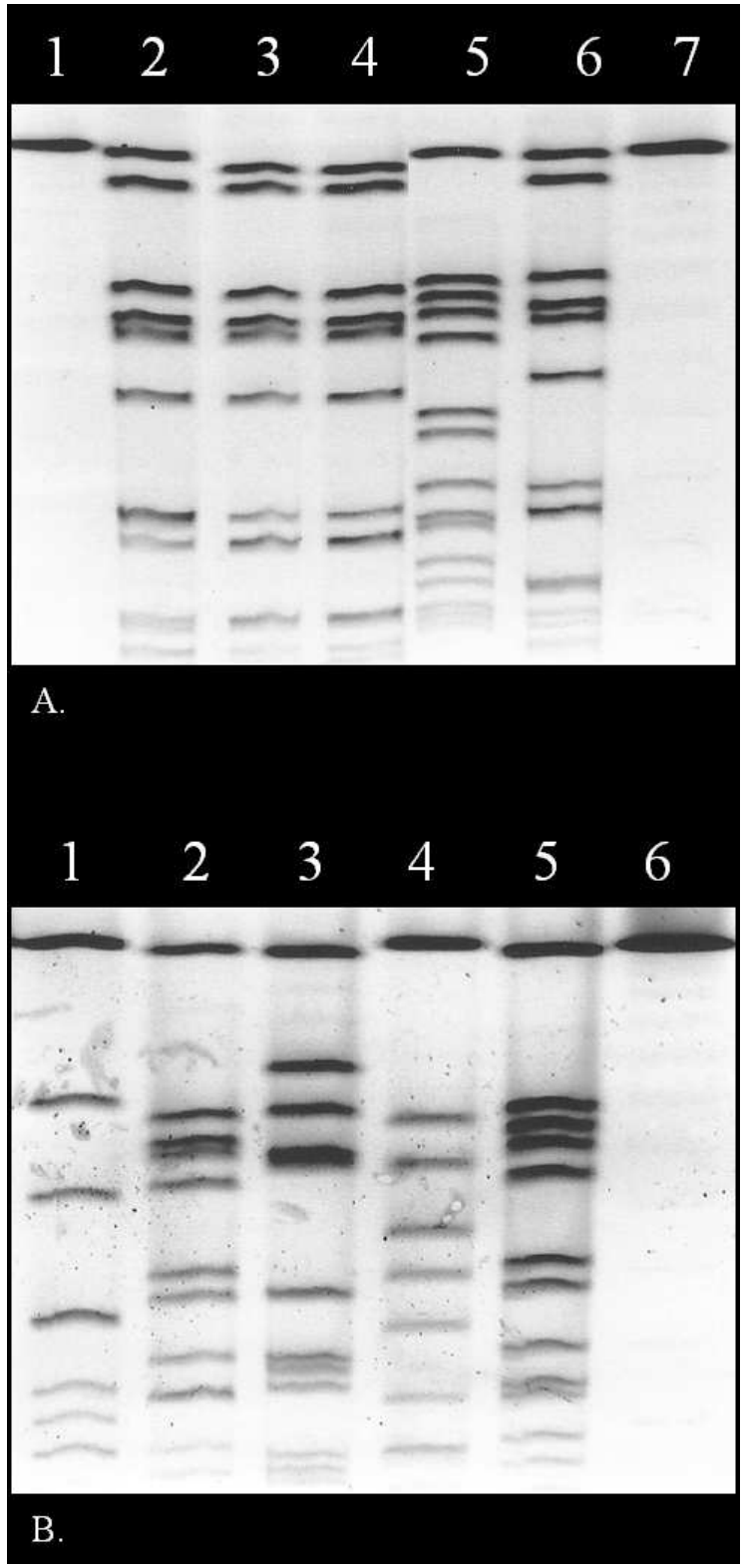

$83 \times 176 \mathrm{~mm}(150 \times 150 \mathrm{DPI})$

John Wiley \& Sons, Inc. 